\title{
NOWCASTING: ANÁLISE DO SISTEMA DE MONITORAMENTO E ALERTA DA DEFESA CIVIL DO ESTADO DE SANTA CATARINA

\author{
André Francisco Pugas ${ }^{1,2}$ and Adriano Vitor ${ }^{2}$
}

\section{Resumo}

Os sistemas de alertas podem ser considerados como uma das principais medidas não estruturais de preparação a desastres naturais. A ocorrência de eventos climáticos extremos estão se tornando cada vez mais comuns, principalmente dentro do contexto catarinense. Diversos estudos indicam que tais fenômenos estão associados com os efeitos das mudanças climáticas e o efeito antrópico no ambiente. Diante dessas premissas, a pesquisa visa analisar como o sistema de alerta de desastres da defesa civil, Nowcasting, realiza a produção dos alertas a população diante os fenômenos hidrometeorológicos. Nesta pesquisa de natureza aplicada, será utilizado uma abordagem qualitativa com o objetivo descritivo do sistema de alerta catarinense utilizado nos procedimentos técnicos documentais; Será identificado quais produtos e serviços meteorológicos estão disponíveis para a emissão dos alertas, quais os critérios são utilizados para decidir a comunicação dos alertas, quais foram os alertas disseminados no período de 2018 a 2021 e a distribuição deles por município. É esperado que esta pesquisa possibilite propostas de melhoria da ferramenta pela comunidade científica após uma análise qualitativa da produção dos alertas e subsidiar aos municípios o conhecimento do risco hidrometeorológico inerente às suas regiões e a disseminação das informações a nível local.

\section{Palavras-chave}

Sistema de alerta, eventos extremos, gestão de risco de desastres.

\footnotetext{
${ }^{1}$ Corpo de Bombeiros Militar de Santa Catarina, Florianópolis, Santa Catarina, Brasil

${ }^{2}$ Programa de Mestrado em Clima e Meio Ambiente, Instituto Federal de Santa Catarina

Corresponding author:

Adriano Vitor, Programa de Mestrado em Clima e Meio Ambiente, Instituto Federal de Santa Catarina

Email:adriano.vitor@ifsc.edu.br.
} 


\section{INTRODUÇÃO}

De acordo com o Ipcc (2021), as mudanças climáticas afetarão diversas regiões do globo, algumas mais do que outras, porém, nenhuma região ficará imune pelos impactos das mudanças climáticas. Esses impactos podem resultar em enormes custos humanos e econômicos que superam em muito os custos das ações de prevenção e preparação.

Em Santa Catarina, segundo Rodrigues (2020), somente no ano de 2020 o Estado foi impactado por ciclones, tempestades, tornados, massas polares e sistemas atmosféricos. Tais eventos foram monitorados por especialistas e meteorologistas de órgãos do poder público, como Epagri/CIRAM e Defesa Civil, com o objetivo de compreender os fenômenos ocorridos e tomar medidas necessárias à preparação e mitigação dos produtos deletérios resultantes desses fenômenos.

A Organização das Nações Unidas (ONU), por meio do objetivo de desenvolvimento sustentável (ODS) número 11 define que até 2030, é necessário reduzir significativamente o número de mortes e pessoas afetadas por desastres naturais de origem hidrometeorológica e climatológica, além de diminuir as perdas econômicas diretas causadas por esses desastres (Onub, 2015).

A Organização Mundial de Meteorologia (WMO) preconiza a necessidade de reduzir a perda de vidas e bens causadas por desastres naturais e outras catástrofes relacionadas ao clima, além de preservar o meio ambiente e buscar o equilíbrio no clima global para as gerações futuras de maneira sustentável, por meio de sistemas integrado de monitoramento e distribuição de dados hidrometeorológicos (WMO,2019).

O Marco de Sendai (Onua, 2015) estabelece a colaboração entre a comunidade acadêmica, instituições públicas, privadas e comunidade em geral a níveis globais e regionais para desenvolver instrumentos e ferramentas para redução do risco de desastres de maneira significativa para as mudanças climáticas, proteção a biodiversidade, desenvolvimento sustentável, erradicação da pobreza, meio ambiente, agricultura, saúde.

Neste sentido, os sistemas de alerta de desastres naturais, específicos ou multi ameaças, são desenvolvidos justamente para realizar o monitoramento das condições climáticas e ambientais com potencial deflagrador de algum tipo de desastre e alertar a população ou públicos específicos ao qual tenham interesse em tais informações como os setores do agronegócios, forças de segurança e defesas civis municipais (UNISDR, 2006).

Segundo Heffer (2013) diversas cidades têm destinado investimentos consideráveis no desenvolvimento de sistemas de alerta para eventos climáticos extremos na América Latina, devido ao potencial que estas ferramentas apresentam em preparar as pessoas e retirá-las do perigo em tempo hábil. Os severos danos e perdas de vidas humanas são mitigados quando um sistema de alerta é implementado adequadamente em se tratando de eventos extremos.

Embora a disponibilidade dos serviços meteorológicos tenha aumentado, ao mesmo tempo que o número de instituições de monitoramento, eventualmente, as informações sobre riscos e prevenção não chegam a toda população. Neste sentido, há necessidade de estratégias e instrumentos que possibilitem a comunicação entre a população em situação de risco e gestores públicos (SORIANO e HOFFMANN, 2016).

É preciso fortalecer o conhecimento científico sobre a climatologia local, por meio de pesquisas sistemáticas, observação do sistema climático e dos sistemas de alertas de desastres, de forma que informações geradas por esses serviços apoie a redução do risco de desastres e a tomada de decisão na disseminação dos alertas de desastres (CALSTER; REINS, 2021). 
No ano de 2016, por meio de um projeto piloto desenvolvido em parceria com a Agência Nacional de Telecomunicações (ANATEL) e a Defesa Civil do Estado de Santa Catarina, foi criado o Nowcasting. "É um serviço gratuito de envio de mensagens de alerta de riscos de desastres e emergências por SMS, serviço de mensagens curtas disponíveis aos aparelhos telefônicos móveis (SANTACATARINA, 2020).

Diante disso, o objeto de pesquisa deste trabalho é analisar como ocorre o processo dos alertas de desastres de origem hidrometeorológicos emitidos pelo Nowcasting da Defesa Civil no Estado de Santa Catarina? Para isso, a pesquisa buscará identificar os produtos e serviços meteorológicos disponíveis para a emissão dos alertas hidrometeorológicos e como ocorre o processo decisório para emissão dessa informação à população. Também será realizado o levantamento dos alertas hidrometeorológicos emitidos no período de 2018 a 2021, verificando a distribuição dos alertas por cidades e regiões.

\section{REVISÃO BIBLIOGRÁFICA}

Atualmente, existem diversas definições de desastres na literatura nacional e internacional. Contudo, até o momento não existe um conceito universal de desastre o que prejudica a compreensão dos fenômenos e os debates científicos sobre o tema, assim, gerando inúmeras dúvidas sobre quais fenômenos podem ser associados a determinada definição. (FAVERO et al., 2014).

Segundo a United Nation Office for Disaster Risk Reduction (UNDRR, 2019) os desastres podem ser definidos como uma perturbação do funcionamento de uma comunidade ou sociedade de maneira severa independente da escala. As condições de exposição, vulnerabilidade e capacidade dessas comunidades associado a interação de eventos perigosos acarretam em perdas e impactos humanos, materiais, econômicos e ambientais.

Ainda assim, o desastre é o resultado da combinação de ameaças/perigo, condições de vulnerabilidade e da insuficiente capacidade ou medidas para reduzir as consequências negativas e potenciais do risco (Trajber, Olivato, \& Marchezini, 2017). Em resumo, um desastre ocasiona danos e prejuízos às pessoas de maneira direta e indireta. Atinge as fontes de alimentação, água, saúde (meio ambiente), moradias, transportes, hospitais (infraestrutura) devido ao impacto de uma ameaça que ultrapassa a capacidade local de responder e atender as consequências de maneira adequada.

Diversos pesquisadores acreditam que as mudanças climáticas estão influenciando a ocorrência e impacto de desastres, principalmente porque o número e a gravidade dos desastres tem aumentado em todo o mundo (Bouwer, 2010).

As mudanças climáticas ocorrem devido às variações naturais de baixa frequência, e por causa da alta concentração constituintes de poluição atmosférica por meio de emissões antropogênicas de gases de efeito estufa. O problema evoluiu consideravelmente nos últimos anos a ponto de se tornar uma questão ambiental de grande relevância (Ipcc, 2021).

As percepções sobre o atual cenário das mudanças climáticas que estão ocorrendo, remete a reflexão se tais efeitos são resultantes da forças naturais ou forçantes antropogênicas e se essas mudanças afetam a frequência, intensidade ou a duração de vários desastres naturais (BLANK, 2015).

De acordo com Phillips et al. (2015) as opiniões sobre as mudanças climáticas bem como os efeitos potenciais sobre os desastres naturais são inferidas pelo conhecimento tanto das mudanças climáticas quanto dos desastres naturais. Deste modo, o nível de conhecimento sobre qualquer assunto pode ser parametrizado pela perspectiva da população sobre a relação entre mudanças climáticas e desastres naturais. 
Recentemente, no ano de 2021, chuvas extremas na Alemanha causaram diversas mortes por inundação, considerada uma das mais devastadoras da história moderna da região europeia. Pesquisas iniciais sugerem que o evento foi causado por tempestades consideradas extremas que se moviam lentamente sobre a Europa central (Zscheischler, 2021). As mudanças climáticas contribuíram para acelerar a evaporação e tem como forte hipótese aumentar a probabilidade de tempestades lentas (Kahraman, Kendon, Chan, \& Fowler, 2021).

De acordo com Herring et al. (2018) os eventos extremos são condições meteorológicas e climáticas excepcionalmente severas com potencial impactos devastadores na sociedade e nos ecossistemas agrícolas e naturais. Os eventos meteorológicos extremos, em regra, têm curta duração e podem ser exemplificados como ondas de calor, congelamentos, chuvas torrenciais, tornados, ciclones tropicais e inundações (Walsh et al., 2014).

Contudo, Lindsey (2016) explica que eventos climáticos extremos perduram por mais tempo do que os eventos meteorológicos ou emergem do acúmulo de eventos meteorológicos que persistem por um período mais longo de tempo. Neste sentido, a seca resultante de longos períodos de precipitação abaixo do normal ou a eclosão de incêndios florestais quando um período prolongado de calor e seca segue uma estação do ano de crescimento anormal úmida e produtiva.

Os desastres naturais estão cada vez mais presentes no cotidiano das comunidades catarinenses nas últimas décadas. A frequência de eventos extremos aumentaram substancialmente juntamente com os danos e prejuízos ocasionados pelos impactos cada vez mais intensos. Dos dez (10) municípios mais atingidos por desastres no Brasil no período de 1991 a 2012, oito (8) municípios estão localizados em Santa Catarina: Abelardo Luz, Canoinhas, Chapecó, Concórdia, Florianópolis, Joinville, Tangará e Seara (UFSC/CEPED, 2013).

O Estado apresenta um grande número de eventos extremos e também pelo maior número de pessoas atingidas. Dentre os quase 5.500 municípios do País, 70 são considerados os de maior incidência de danos e dentro desse universo, o Estado catarinense contabiliza 34 municípios dentro dessa triste estatística (CARDOSO et al. 2014). Grande parte de tais eventos extremos podem ser associados resultante das mudanças climáticas.

Apesar dos impactos ocasionados pelos eventos extremos serem cada vez evidentes, as metodologias para adaptar-se a esses impactos, o grau de nossa compreensão e capacidade de previsão variam amplamente entre os diferentes setores e disciplinas (Sillmann, Sippel, \& Russo, 2019).

Em consonância com a gestão dos riscos de desastres, os processos de informação, previsão, comunicação e educação têm prestado suporte às políticas preventivas de maneira que as pessoas convivam com o risco. Há uma tendência a promover e disseminar conhecimentos técnico-científicos que possibilitem compreender os fenômenos naturais e identificar áreas de risco, bem como propor ações de prevenção e resposta para reduzir os impactos de eventos extremos (SULAIMAN;ALEDO, 2016).

De acordo com United Nations Office for Outer Space Affairs (UNOOSA, 2020) é necessário haver a colaboração através de produtos de informação como monitoramento via satélite, e outras tecnologias disponíveis para gestão de desastres. A combinação desses recursos de observação do planeta realizado por diferentes agências, permite o desenvolvimento de novas tecnologias e experiência integrada para coordenar medidas para responder rapidamente a grandes desastres.

Como uma das formas de prevenção de risco a desastres é fundamental o desenvolvimento de sistemas de alerta para eventos extremos. Estes sistemas têm a função de dar publicidade à informações do andamento de uma possível ameaça a população, como também quais são as medidas adequadas que 
as pessoas devem adotar para promover sua autoproteção de forma mais segura e que gere menos danos ou prejuízos (LUZ; FILGUEIRA, 2018).

De acordo com Basher (2006) o termo sistema de alerta é utilizado multidisciplinarmente, para representar o fornecimento de informações sobre uma condições de perigo iminente, com vistas a adotar medidas para reduzir os riscos envolvidos de maneira antecipada.

Atualmente, são reconhecidos diversos tipos de sistemas de alerta: geofísicos, biológicos, sóciopolíticos, industriais, riscos para a saúde pessoal e muitos outros riscos relacionados. Sorensen (2000) reconhece que os sistemas de alertas são complexos devido ao envolvimento multidisciplinar do conhecimento e das instituições e públicos envolvidos. Geralmente envolvem os campos da ciência, governo, engenharia, iniciativa privada, tecnologia de informação, mídias televisivas digitais e a população.

Dentro desse processo existem diversos tipos de dados, informações, conhecimento, especialistas, stakeholders, profissionais, políticos, e cidadãos envolvidos. O monitorando das ameaças e a tomada a decisão em relação a emissão dos alertas de potenciais riscos de desastres, normalmente são realizadas em salas de controle, operada por profissionais qualificados para analisar as variáveis ambientais, reconhecer as ameaças e vulnerabilidades, e disseminar os alertas como medida de preparação e resposta de emergência (HORITA, et al., 2018).

A UNDRR (2019) caracteriza os sistemas de alertas de desastres como um sistema integrado de monitoramento de ameaças, previsão e avaliação de risco de desastres. Além de realizar a comunicação das informações e preparar as pessoas, comunidades, governos, empresas a adotarem medidas oportunas para reduzir os riscos de desastres na iminência de eventos deletérios.

Ainda assim, os sistemas de alerta estabelecem atividades além da compreensão dos riscos existentes como as de monitoramento e alerta. Quando se fala em monitoramento nos sistemas de alerta, os serviços meteorológicos são o que mais frequentemente vem à mente e essa infra estrutura fornece as previsões para a produção dos alertas (UNDP, 2018).

O monitoramento de potenciais ameaças é realizado por meio da coleta de dados e informações para identificar condições propícias para a instalação de iminentes riscos. Este monitoramento visa fundamentar a disseminação de alertas antecipados de possíveis ocorrência de desastres (Marchezini et al., 2017).

Da mesma forma, os dados e informações quer sejam em relação aos aspectos físicos, quer sejam associados à meteorologia, são analisados para a caracterização dos alertas como acumulados de precipitação, descargas atmosféricas e temperatura do mar. Porém, é necessário também o acréscimo de aspectos de vulnerabilidade, os quais não são objeto de monitoramento por parte dos sistemas de alerta (KELMAN;GLANTZ, 2014).

Neste sentido, a UNDP (2018) define que o monitoramento é o ato de coletar informações em conjunto as variáveis relacionadas ao risco, como chuva ou ondas sísmicas, feito por meio de observações diretas. Entretanto, tais observações apresentam certa limitação devido ao ambiente caótico inerente à proximidade das ameaças meteorológicas e limitando também a adoção de ações de redução derisco.

O termo previsão é muitas vezes considerado como uma expressão qualitativa de probabilidade com o objetivo de determinar a responsabilidade ou culpa quando o evento crítico se instala. Isso se aplica claramente a desastres, o que remete a importância dos serviços de monitoramento nos sistemas de alerta (KELMAN;GLANTZ, 2014). 
Segundo Basher (2006) os meteorologistas utilizam o termo End to End para os sistemas de alerta que realizam previsões e avisos mais relevantes e utilizáveis para a população em geral. Sistemas de alerta baseados no método End-to-End tem como objetivo avaliar e gerenciar as ameaças por meio de previsões como os perigos hidrometeorológicos, geológicos e eventos extremos, associados às mudanças climáticas e variabilidade climatológica (UNOOSA, 2020).

No Brasil, o Centro Nacional de Gerenciamento de Riscos e Desastres (CENAD) é responsável pela sistematização dos alertas hidrometeorológicos e de riscos de movimentos de massa.Também realiza a disseminação de boletins informativos sobre as possibilidades de eventos extremos (Brasil, 2021).

O CENAD analisa e gerencia essas informações com o objetivo de assistir os estados e municípios do Brasil nas ações de preparação para desastres junto às comunidades mais vulneráveis. Este órgão é coordenado pela Secretaria Nacional de Proteção e Defesa Civil (SEDEC) e atua 24 horas por dia no monitoramento de possíveis desastres no país (HORITA et al., 2017).

Ao mesmo tempo, de acordo com Marchezini et al. (2017), o Centro Nacional de Monitoramento e Alertas de Desastres Naturais (CEMADEN) é responsável a nível federal por realizar o monitoramento de desastres relacionados a enxurradas e deslizamentos, e ainda, realiza de forma compartilhada o monitoramento de ameaças por outras agências federais: como a Agência Nacional de Águas (ANA) em relação aos dados hidrológicos e o Serviço Geológico do Brasil (CPRM).

A Política Nacional de Proteção e Defesa Civil (PNPDEC) prevê que as ações de proteção e defesa civil sejam integradas e contínuas, no monitoramento dos eventos meteorológicos, hidrológicos, geológicos, biológicos, nucleares, químicos e outros potencialmente causadores de desastres. Além de realizar a produção de alertas prévios sobre a possibilidade de ocorrência de desastres naturais. (BRASIL, 2012).

A integração dessas ações representa a gestão colaborativa para a redução dos riscos de desastres no Brasil e define a composição do Sistema Nacional de Proteção e Defesa Civil (SINPDEC) com diferentes órgãos públicos federais, estaduais e municipais.

Entretanto, em Santa Catarina, a partir de janeiro de 2017, os alertas de desastres são comunicados por meio de serviço de mensagens curtas (SMS) pela Defesa Civil Estadual. Este projeto foi aderido por alguns estados nos anos de 2018 e 2019 como Espírito Santo, Sergipe, Mato Grosso do Sul, Goiás, Rio de Janeiro, São Paulo, Rio Grande do Sul, Paraná e Distrito Federal (BRASIL, 2018).

Os serviços de alerta por SMS informam sobre a incidência de fenômenos meteorológicos relacionados a inundações, deslizamentos de terra, granizo e tempestades de vento para os usuários deste serviço gratuito. O usuário realiza a adesão do serviço enviando uma mensagem de texto para o número 40199 para receber o SMS gratuitamente, informando o Código Postal do local de interesse (Saito, Lima, \& Dias, 2019).

A Defesa Civil Estadual conta com o Centro Integrado de Gerenciamento de Riscos e Desastres do Estado (CIGERD), localizado na capital do Estado, Florianópolis. Neste local é realizado o monitoramento, previsão e disseminação dos alertas onde é possível visualizar, em tempo real, os níveis de pluviosidade em determinadas regiões, focos de incêndio, velocidade dos ventos, a umidade relativa do ar e também a temperatura média (Vazquez \& Guimarães, 2018). A figura abaixo demonstra sucintamente o sistema de alerta em Santa Catarina.

O processo integrado dos dados e informações produzidas pelo CIGERD pode determinar o lançamento de um alerta. Profissionais de diversas áreas, como meteorologistas, hidrólogos, geotécnicos e militares realizam presencialmente a análise dos dados 24 horas por dia. 


\section{METODOLOGIA}

Nesta pesquisa de natureza aplicada, será utilizado uma abordagem qualitativa com o objetivo de descrever o sistema de alerta utilizado pela Defesa Civil do Estado de Santa Catarina por meio de procedimentos técnicos documentais.

Inicialmente, será realizado o levantamento dos produtos tecnológicos disponíveis à Defesa Civil para realizar o monitoramento das condições climáticas. Esse levantamento será feito por meio de consulta aos profissionais atuantes no monitoramento, meteorologistas e técnicos responsáveis pelo setor. Também será realizada a pesquisa in loco da estrutura disponível, realizando registros fotográfícos e a descrição dos equipamentos e tecnologias disponíveis.

Em seguida, será realizada uma pesquisa documental nos procedimentos, processos e protocolos vigentes na Defesa Civil, com o objetivo de compreender como ocorre o processo decisório da emissão ou não de um alerta para determinada região diante os fenômenos hidrometeorológicos. Na sequência, será realizado a consulta aos profissionais responsáveis por essa etapa, descrevendo como o processo é desenvolvido para cada um dos fenômenos hidrometeorológicos.

Posteriormente, serão catalogados os alertas de fenômenos hidrometeorológicos emitidos no período de 2018 a 2021. O banco de dados utilizados para tal pesquisa será da Agência Nacional de Telecomunicações (ANATEL), devido os alertas serem emitidos por meio da interface de divulgação de alertas públicos (IDAP) utilizado pela Defesa Civil para a comunicação dos alertas por SMS e TV. E ainda, será catalogado quais foram a natureza dos alertas emitidos dentro do período estipulado, conforme a classificação dos desastres naturais estabelecido pelo Código Brasileiro de Desastres (COBRADE).

Após o levantamento dos dados dos alertas, emitidos pela Defesa Civil por meio do IDAP disponível pela ANATEL, será realizada a classificação de quais alertas de origem hidrometeorológica foram emitidos no período de 2018 a 2021, por cidades; Para essa classificação, será utilizado o COBRADE, como elemento padrão para caracterizar o fenômeno hidrometeorológico.

A apresentação desses alertas será demonstrada por meio de tabelas e gráficos representando a quantidade de alertas emitidos a cada cidade e quais fenômenos hidrometeorológicos as afetaram.

\section{REFERÊNCIAS}

A, W. (2019). Basic documents no. 1. world meteorological organization (Vol. 15). Geneva, Switzerland, V.

Basher, R. (2006). Global early warning systems for natural hazards: systematic and people-centred. Philosophical Transactions of The Royal Society A. $n, 364,2167-2182$.

Blank, D. M. P.(n.d.). Retrieved from http: / / doi .org/10.4215/RM2015.1402.0010

Bouwer, L. M. (2010). Disasters and climate change: analyses and methods for projecting future losses fromextreme weather. 144 p. Tese, Institute for Environmental Studies (IVM), Amsterdã, Holanda.

Brasil. (2021).

Calster, G. V., \& Reins, L. (2021). The paris agreement on climate change: A commentary. Cheltenham, UK: Edward Elgar. 
Cardoso, D., \& Enchentes. (2013). deslizamentos e a sociedade em rede: um estudo sobre o fluxo de informação em desastres naturais a partir do caso de petrópolis. Florianópolis: Mestrado em Ciência da Informação.

Catarina, S., \& Civil, D. (2021). Nowcast - alerta sms. Santa Catarina. Retrieved from <https: / / www.defesacivil.sc.gov.br/alertas-sms/>.Acessoem

de Proteção e Defesa Civil PNPDEC. Brasília, B. P. N. (Ed.). (2012). Lei federal $n^{o}$ 12.608. presidência da república. Retrieved from http: //www.planalto.gov.br/ccivil_03/ ato2011-2014/2012/lei/112608.htm

Favero, E., Sarriera, J. C., \& Trindade, M. C. (n.d.). Desastre na Perspectiva Sociológica e Psicológica. Psicologia em Estudo, 19(2), 201-209.

Heffer, C. O. (2013). Rio de Janeiro City's Early Warning System for Heavy Rain. Practical Action Consulting, 1-7.

Herring, S. C., Christidis, N., Hoell, A., Kossin, J. P., Schreck, C. J., \& Stott, P. A. (2018). American Meteorological Society.

Horita, F. E., Albuquerque, J. P. D., Marchezini, V., \& Mendiondo, E. M. M. (n.d.). Retrieved from https://doi.org/10.1016/j.dss.2017.03.001

Ipcc, A. (2021). Climate change 2021: The physical science basis. contribution of working group $i$ to the fourth assessment report of the intergovernmental panel on climate change. Cambridge, Reino Unido: Cambridge University Press.

Kahraman, A., Kendon, E. J., Chan, S. C., \& Fowler, H. J. (2021). Quasi-stationary intense rainstorms spread across Europe under climate change. Geophysical Research Letters. v, 48, 1-11.

Kelman, I., \& Glantz, M. H. (2014). Retrieved from https: / / doi .org/10 . 1007/978-94-017 $-8598-35$

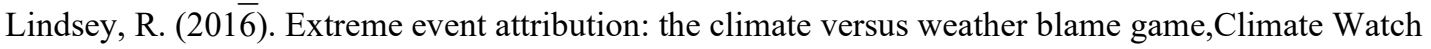
Magazine, NOAA, 15 Dez. Disponivel em, 12-12.

Luz, M. B., \& Filgueira, H. J. A. (2018). Sistemas de alerta a desastres relacionados com fenômenos naturais: algumas alternativas no brasil e no mundo. In: I Encontro Nacional de Desastres. Associação Brasileira de Recursos Hídricos. Disponível em, 23-23.

Marchezini, V., Londe, L. R., Bernardes, T. ., Ev, C. R., Santos, E. V., Saito, S. M., . . Gonçalves, D. A. (2017).

Onua. (2015). United Nations Office for Disaster Risk Reduction. In UN world conference on disaster risk reduction.

Onub. (2015). Retrieved from <https://nacoesunidas.org/wp-content/uploads/ 2015/10/agenda2030-pt-br.pdf>Acessoem01Ago.2021

Phillips, M. C. K., Cinderich, A. B., Burrell, J. L., Ruper, J. L., Will, R. G., \& Sheridan, S. C. (2015). The effect of climate change on natural disasters: A college student perspective. weather climate and society. Ohio. v.7.

Rodrigues, M. L. G. (2020). Retrieved from https: / / publicacoes.epagri.sc.gov.br/ RAC/article/view/1082. Acessoem

RS e ES passam a contar com serviço de alerta de desastres naturais por SMS. Brasília. (2018). Disponivel em, 15-15.

Saito, S. M., Lima, G. R. T., \& Dias, M. C. A. (2019). Evaluation by the end-users of disaster risk warnings in brazil. Ago. Retrieved from https://doi:10.18472/SustDeb.v10n2 


\section{.2019 .24908}

Sillmann, J., Sippel, S., \& Russo, S. (2019). Climate extremes and their implications for impact and risk assessment. 1 ed. Oxford, Reino: Elsevier. Retrieved from https://doi.org/10.1016/ C2017-0-01794-9

Sorensen, J. H. (2000). Hazard warning systems: Review of 20 years of progress. Natural Hazards Review. v, 1(2), 1527-6988.

Soriano, E., \& Hoffmann, W. (2016). The evolution of the protection systems against natural disasters in Brazil: laws, agencies, information and knowledge. In Management of Natural Disasters (Vol. 150, p. 49-58). WIT Press.

Sulaiman, S. M., \& Aledo, A. (2016). Dilemas ambientais e fronteiras do conhecimento. Estudos Avançados. v. 30, n. 88. Set-Dez, 11-23.

Trajber, R., Olivato, D., \& Marchezini, V. (2017). Conceitos e Termos para a Gestão de Riscos de Desastres na Educação. CEMADEN. 03 Abr. Disponível em.

United Nation Office for Outter Space Affairs. (2020). UN-SPIDER Newsletter. v, 1, 26-26.

United Nations Development Programme. Five approaches to build functional early warning systems. (2018). UNDP, 67-67.

United Nations Office For Disaster Risk Reduction. (2006). Early Warning - From Concept to Action: the Conclusions of the Third International Conference on Early Warning (EWC III). Bonn. Alemanha. $10 \mathrm{p}, 27-29$.

United Nations Office For Disaster Risk Reduction. Terminology - Early Warning System. (n.d.). Disponível em.

Vazquez, F., \& Guimarães. (2018). Alertas de desastres socioambientais no estado de santa catarina sob a perspectiva da ciência da informação. 2018. 155 p.dissertação (mestrado) - universidade federal de santa catarina, centro de ciências da educação, programa de pós graduação em ciência da informação. Florianópolis.

Walsh, J., Wuebbles, D., Hayhoe, K., Kossin, J., Kunkel, K., Stephens, G., . . Somerville, R. (2014). CHAPTER 2 OUR CHANGING CLIMATE. Climate Change Impacts in the United States: The Third National Climate Assessment. Out, 19-67.

Zscheischler, J. (2021). Retrieved from https://doi.org/10.1016/j.wace.2021.100381

\section{Author Biographies}

André Francisco Pugas Discente do Programa do Mestrado em Clima do Instituto Federal de Santa Catarina.

Adriano Vitor Docente do Programa de Mestrado em Clima e Meio Ambiente do Instituto Federal de Santa Catarina 